\title{
Auralization of Amplitude Modulated Helicopter Flyover Noise
}

\author{
Siddhartha Krishnamurthy ${ }^{1}$ and Stephen A. Rizzi ${ }^{2}$ \\ NASA Langley Research Center, Hampton, VA 23681, USA
}

\begin{abstract}
Rotorcraft noise is an active field of study as the sound produced by these vehicles is often found to be annoying. A means to auralize rotorcraft flyover noise is sought to help understand the factors leading to annoyance. Previous work by the authors auralized a complete flyover event in which the source noise synthesis traversed a range of emission angles. The source noise definition process for the synthesis used helicopter flyover recordings. Although this process yielded both periodic and aperiodic (modulation) components at a set of discrete emission angles, only the periodic components were used in the previous work to synthesize the sound of the flyover event. In the current work, aperiodic amplitude modulation is incorporated into the source noise synthesis to improve its fidelity toward assessing rotorcraft noise annoyance. The method is demonstrated using ground recordings from a flight test of the AS350 helicopter for the source noise definition.
\end{abstract}

\section{Nomenclature}

$A \quad=$ harmonic amplitude $(\mathrm{Pa})$

$f \quad=$ frequency $(\mathrm{Hz})$

$\phi_{o} \quad=$ harmonic phase $(\mathrm{rad})$

$M \quad=$ number of tail rotor harmonics

$N=$ number of main rotor harmonics

$p \quad=$ pressure $(\mathrm{Pa})$

$t \quad=$ time $(\mathrm{s})$

$\theta \quad=\operatorname{polar}($ elevation) angle

\section{Introduction}

$I^{N}$

$\mathrm{N}$ recent years, helicopter use in both the private and commercial sectors has seen an increase around major U.S.

cities. With an increase in usage, comes an increase in noise, which can lead to increased annoyance. Annoyance has been the basis for several actions across the U.S. to limit helicopter operations ${ }^{1,2}$ and, consequently, is a focus of research by many in the rotorcraft community.

Factors that lead to annoyance can fall under two main categories: acoustic and nonacoustic. An example of an acoustic factor is the impulsiveness of a sound. Age, fear and noise sensitivity are nonacoustic factors that can contribute to an individual's evaluation of a sound and its annoyance, ${ }^{3}$ e.g., the association of a helicopter as an unsafe device. The present effort is directed at the long-term goal of producing high-fidelity auralizations to help understand the acoustic factors leading to annoyance. Auralization is a technique for creating audible sound files from numerical data. ${ }^{4}$ Here, it refers to the combined processes of source noise synthesis and propagation to a ground observer. Attaining this long-term goal will require the generation of sounds from vehicles and operating conditions for which recordings do not exist.

An auralization method for rotorcraft fly-in noise with sound synthesis at a single low emission angle based on recorded flyover data was previously developed for use in audibility studies. ${ }^{5}$ Although the flyover recording contained aperiodic fluctuations, or modulations, in the sound pressure, the source noise synthesis purposefully omitted these modulations in the source and propagation path in order to simplify interpretation of results from sound jury audibility testing. ${ }^{6}$ Omitting the modulations was made possible by using a source noise definition that generated separate main and tail rotor pressure time histories for a single blade passage. ${ }^{7}$ For each rotor, unsteadiness of the

${ }^{1}$ Research Engineer, Structural Acoustics Branch, Member AIAA

${ }^{2}$ Senior Researcher for Aeroacoustics, Aeroacoustics Branch, Fellow AIAA 
source was removed by applying synchronous time averaging of multiple blade passages. The propagation model used also neglected turbulence, thereby removing an additional potential source of sound pressure fluctuations.

It is possible that fluctuations in the noise play a role in community acceptance of vertical lift vehicles. In order to study the effects of such fluctuations, future sound jury testing will need high-fidelity rotorcraft source noise synthesis that includes representative aperiodic modulations. Although the causes of the aperiodic modulations are not completely understood, they occur within different steady flight operating conditions and during flight maneuvers.

Previous efforts have sought to develop approaches to synthesize source noise using periodic and aperiodic components. Hardwick ${ }^{8}$ adapted a method originally developed by Allen et al. ${ }^{9}$ for separating the aperiodic components from the periodic components of turbofan tonal noise to rotorcraft harmonic noise. Hardwick's approach decomposed segments of de-Dopplerized recorded noise into periodic and aperiodic amplitude and phase components, enabling reconstruction of the original waveform signal with and without aperiodic components. The recorded noise was from a steady level flight. Therefore, the aperiodic components in this case were not due to purposeful maneuvering. Through human subject testing, he found that the subjects could differentiate reconstructed signals containing amplitude and phase modulation components from those containing only the periodic components. ${ }^{10}$ However, this approach was only able to reconstruct the recorded time segment for which modulation data were extracted.

Pera et al. ${ }^{11}$ extended Hardwick's method to allow synthesis of signals of indefinite duration through the development of an amplitude modulation model at each emission angle. The model consisted of filtered white noise with model parameters being the gain and corner or cutoff frequency of the filter. Although Pera et al. demonstrated the synthesis of a helicopter flyover over a range of emission angles, this synthesis process only used the periodic components of the source noise signal. The amplitude modulation model was only extended for a stationary source and observer, and was not successfully extended for a full flyover with a moving source.

The long-term goal of the current effort is to extend auralization capabilities to permit the generation of a full flyover such that it retains unsteadiness or modulations characteristic of the source. This paper applies the amplitude modulation model developed by Pera et al. toward the aperiodic synthesis of a complete helicopter flyover. This is done by interpolating the parameters of the amplitude modulation model over the flyover duration and using an overlap-add technique to sum the time-domain output. Determining the model parameters will be discussed in this paper. As an additional improvement over Pera et al., this paper will apply phase alignment and spherical phase interpolation to the periodic portion of the synthesis. The resulting synthesized source noise will be propagated to a ground observer using the propagation capabilities of the NASA Auralization Framework (NAF). ${ }^{12}$

The same recorded AS350 helicopter flight test data used by Pera et al. will again be used to determine the periodic and aperiodic components. ${ }^{13,14}$ As in the earlier works, this paper only considers tonal noise from the main and tail rotors and does not consider other noise sources on the vehicle. Section II describes how the periodic and aperiodic signal parameters are extracted and how additive synthesis can reconstruct the recorded signal. Section III covers the de-Dopplerization and segmentation method from Greenwood and Schmitz ${ }^{7}$ to separate the main and tail rotor signatures. Section IV describes the amplitude modulation model to extend the aperiodic amplitude parameter indefinitely in time. Section V combines all the elements into an auralization of flyover noise with amplitude modulation, and compares that with the recorded signal on which the auralization was based.

\section{Source Noise Decomposition}

This section summarizes a method for decomposing a helicopter flyover recording into amplitude, frequency, and phase components for the main and tail rotor signals. Source characteristics of a vehicle are assumed to change as a function of emission angle. If recordings of a moving helicopter were available at different fixed emission angles, decomposing these stationary recordings could be used to define angle-dependent characteristics. Since such recordings are unavailable, it will be assumed that periodic signal components can be determined by analyzing finite time segments of a flyover recording. The coarseness of the segmentation will determine how well changes to the source characteristics are captured in the final auralization. Although aperiodic components vary temporally and spatially, the signal decomposition method assumes they vary only temporally with each segment.

\section{A. Decomposition and reconstruction}

After decomposing each time segment of a helicopter flyover recording and extracting the rotor signal components, the original segments can be reconstructed to test the performance of the decomposition and extraction process. Reconstruction of the combined main and tail rotor pressure time histories for a recording segment is performed in the time domain using an additive synthesis approach, ${ }^{5}$ that is, 


$$
p_{q}(t)=\sum_{i=1}^{N} A_{i, q}^{M R}(t) \cos \left(\phi_{i, q}^{M R}(t)\right)+\sum_{i=1}^{M} A_{i, q}^{T R}(t) \cos \left(\phi_{i, q}^{T R}(t)\right)
$$

in which the subscript $q$ represents the segment number, the subscript $i$ represents the $i$ th harmonic of either the main or tail rotor blade passage frequency (BPF), A and $\phi$ are the amplitudes and phases of each harmonic, $N$ and $M$ are the number of main and tail rotor harmonics, respectively, and the superscripts $M R$ and $T R$ denote main and tail rotor, respectively. The amplitudes and phases of each harmonic in Eq. (1) can be expanded to

$$
A_{i, q}(t)=\bar{A}_{i, q}+\tilde{A}_{i, q}(t)
$$

and

$$
\phi_{i, q}(t)=2 \pi \bar{f}_{i, q} t+\tilde{\phi}_{i, q}(t)+\phi_{o, i, q}
$$

in which $\bar{f}_{i}$ is the $i$ th harmonic frequency, which is constant in time, $\bar{A}$ and $\phi_{o}$ represent periodic amplitude and phase components, respectively, that are assumed constant for the duration of the segment recording, and the tilde quantities represent aperiodic (modulation) components. ${ }^{8}$ Note that the relationship between the phase modulation and frequency is given by

$$
\tilde{\phi}_{i, q}(t)=2 \pi \int_{0}^{t} \tilde{f}_{i, q}(\tau) d \tau
$$

By dropping the explicit time dependence from the tilde quantities and the elimination of the $M R$ and $T R$ superscripts in Eq. (1), the main and tail rotor harmonic summations can be summarized in the single equation given by

$$
p_{q}(t)=\sum_{i=1}^{N, M}\left[\bar{A}_{i, q}+\tilde{A}_{i, q}\right] \cos \left(2 \pi \bar{f}_{i, q} t+\tilde{\phi}_{i, q}+\phi_{o, i, q}\right) .
$$

Reconstruction of the original signal segment thus amounts to the determination of the quantities on the right hand side of Eq. (5) through a signal decomposition process. Since each recorded segment spans a range of emission angles, the reconstruction is approximated as reconstructing the time history at the segment's center emission angle.

The signal decomposition process used to determine the periodic and aperiodic quantities in Eq. (5) was presented in Pera et al. and will not be described in detail here. It follows the method by Hardwick, ${ }^{8}$ in which the main and tail rotor harmonics from a recording segment are isolated through bandpass filtering. Amplitude modulation of each harmonic is subsequently extracted through a Hilbert transform, and the phase portion is extracted from the argument of the Hilbert transform. Using this extracted phase, a simulated annealing technique is used to estimate the frequency, $\bar{f}_{i} \cdot{ }^{15}$ The quantity $2 \pi \vec{f}_{i} \vec{t}$, for some time vector $\vec{t}$, is subtracted from the extracted phase, and the initial phase is calculated as the mean over time of this difference. Note that the offset of the elements in the vector $\vec{t}$ from zero will affect the value of the initial phase. The residual, after the mean is subtracted from the difference, is the modulated phase. Application of these processes, collectively referred to as the "direct method," are thus used to determine the quantities in Eq. (5). ${ }^{11}$ Once the periodic and aperiodic components of each harmonic for a particular segment are extracted, each segment's signal can be reconstructed using Eq. (5) to check the quality of the signal component extraction.

\section{B. Phase alignment}

During the rotor signal decomposition process for recorded segments, the phase relationship between segments must be maintained. This phase relationship is normally maintained when the periodic data (amplitude, harmonic frequency, and initial phase) at each emission angle are the result of an acoustic calculation, for example, from Farassat's formulation $1 \mathrm{~A}^{16}$ using periodic blade loadings as input. However, the phase relationship between segments is not necessarily maintained in the process of segmenting flyover recording data at different emission angles. Pera et al. described how taking the time offset for each recorded segment into account will properly change the values of the initial phase and thus maintain the phase relationship between segments.

When segmenting a recorded signal in this paper, the timing offsets at the start of each segment relative to the start of the first segment were found. With the phase of a harmonic given by

$$
\phi_{i, q}(\vec{t})=2 \pi f_{i} \vec{t}+\phi_{o, i, q}+\phi_{i, q}(\vec{t})
$$

in which $\vec{t}$ is the vector of time samples for segment $q$ offset relative to the start of the first segment, a more accurate initial phase will be produced by subtracting $2 \pi \bar{f}_{i} \vec{t}$ from Eq. (6) after averaging the phase modulation to zero. Otherwise, the initial phase will be estimated as $2 \pi \bar{f}_{i} t_{q}+\phi_{o, i, q}$ where $t_{q}$ is the start time of segment $q$, meaning the initial phase will be dependent on how the recorded signal is segmented. It must be noted that errors resulting from a 
nonzero average of the phase modulation, however small, will cause some phase misalignment, even when segment timing offsets are used.

\section{Modifications to source noise decomposition}

In this paper, two modifications of the direct method of Pera et al. are made. First, a $15 \mathrm{~Hz}$ bandpass filter was used to isolate each harmonic instead of a $20 \mathrm{~Hz}$ bandpass filter. Second, harmonics were subtracted from the original recorded segment as their components were found. Figure 1 shows a flowchart of the modified method for estimating the frequencies, amplitudes, and phases of the harmonic components. The flowchart begins with the recorded signals for each segment and nominal BPF frequencies around which the $15 \mathrm{~Hz}$ bandpass filters will be centered. In this paper, the nominal BPF frequencies were $20 \mathrm{~Hz}$ and $70 \mathrm{~Hz}$, corresponding to the approximate BPFs of the main and tail rotor, respectively.

The first loop and the subsequent averaging process in the flowchart attempts to improve the estimate of the BPFs by averaging the simulated annealing results for the first harmonics in each segment. When one estimates the frequency, $\bar{f}_{i, q}$, of higher harmonics using simulated annealing, the estimated values will not necessarily be integer multiples of the estimated BPF at $\bar{f}_{1, q}$, so for the present work, all subsequent harmonics were fixed to be integer multiples of the averaged BPFs, that is,

$$
\begin{gathered}
\bar{f}_{1}^{M R, T R}=\frac{1}{Q} \sum_{q=1}^{Q} f_{1, q}^{\prime M R, T R} \\
\bar{f}_{i}^{M R, T R}=i \times \bar{f}_{1}^{M R, T R} .
\end{gathered}
$$

Here, $f_{1, q}^{M R, T R}$ are the estimated first harmonics of the main or tail rotor determined through simulated annealing, $q$ is the segment number, and $Q$ is the number of segments. The term $\bar{f}_{i}$ in Eq. (8) replaces $\bar{f}_{i, q}$ in Eq. (5) to indicate that the harmonic frequencies will be constant over the segments. In this paper, the estimated values of the averaged BPFs computed using Eq. (7) were $19.73 \mathrm{~Hz}$ for the main rotor and $70.03 \mathrm{~Hz}$ for the tail rotor. Although these values can change depending on how many segments are used for the average, the first loop in Figure 1 was run just once for this paper, and the same averaged BPFs, $19.73 \mathrm{~Hz}$ and $70.03 \mathrm{~Hz}$, were used for subsequent signal decompositions that used a different number of segments.

After the BPFs are found using Eq.(7), the second loop in the Figure 1 flowchart performs the signal decomposition process on harmonics of the BPF using the direct method described in Section II. Here, all bandpass filters were centered around nominal frequencies that were integer multiples of the nominal BPF frequencies $(20 \mathrm{and} 70 \mathrm{~Hz})$. After a harmonic was isolated, a set of frequencies found using Eq. (8) were used to obtain the initial and modulated phases and reconstruct the segment signal. When the bandpass filter is used to isolate a harmonic, small contributions from harmonics outside of the $15 \mathrm{~Hz}$ bandwidth are still present in the signal, and therefore, influence the harmonic component amplitude and phase quantities estimates. To correctly account for this extra energy, the synthesized harmonic signal obtained from the frequency, phase, and amplitude estimates is subtracted from the original recorded signal for each segment. If the subtraction step in Figure $1, p_{q}(t)=p_{q}(t)-p_{i, q}(t)$, is not performed, the harmonic contributions will sum incorrectly in the reconstruction.

As in Pera et al., tail rotor harmonics were identified and isolated prior to the main rotors. As a result of the harmonic subtraction step just discussed, components of any main rotor harmonic that were within $7.5 \mathrm{~Hz}$, or half the passband width, of a tail rotor harmonic were attributed to that tail rotor harmonic.

Although the signal decomposition and additive synthesis method described with Eqs. (5), (7), (8), and the flowchart in Figure 1 can reconstruct specific segments of a recording, two additional steps are needed in order to accurately synthesize the source noise associated with a continuous flyover event. The first is to extend the duration of the synthesized signal for as long as needed. This requires application of the amplitude modulation model developed by Pera et al. This model is described in Section IV. The second is to smoothly transition the synthesized signal as emission angle varies over the course of the flyover. This is done through an interpolation process discussed in Section V. 


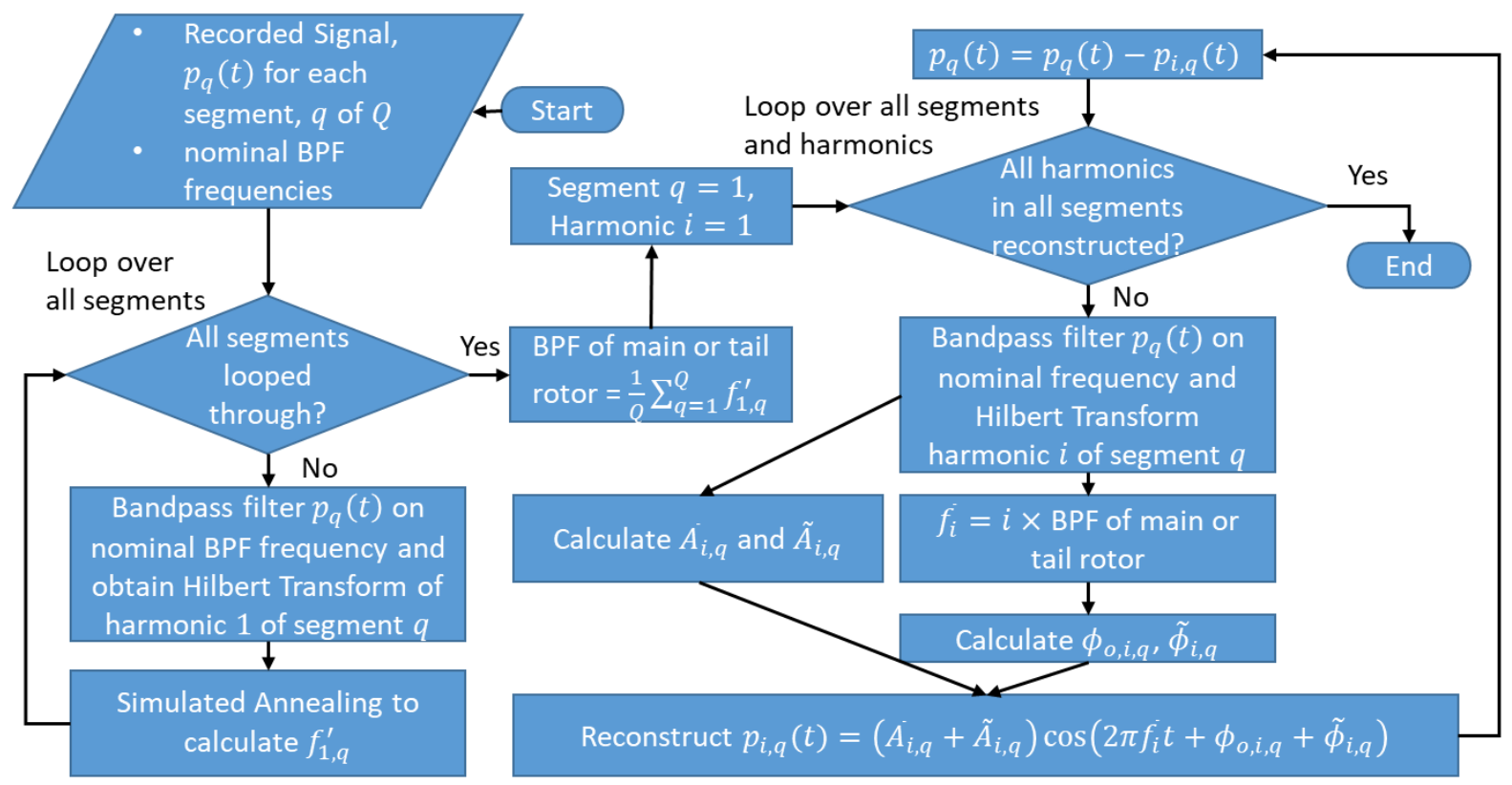

Figure 1. Flowchart for estimating harmonic components.

\section{De-Dopplerized Signal}

In order to determine the source characteristics from a flyover recording, effects of Doppler, atmospheric absorption, ground, terrain, and spreading loss must be removed or mitigated. Here, this is accomplished by a deDopplerization process, which is equivalent to recreating the sound at an observer moving in a fixed radius around the source. The signal decomposition described in Section II is then applied to the result of de-Dopplerization. The black trace in Figure 2 is the original flyover recording of an AS350 helicopter upon which the synthesis and auralization results of this paper are based. In addition to the recorded sound of the flyover, vehicle tracking data was available to determine the vehicle location with respect to a microphone flush with the ground. The azimuthal angle for the flyover recorded here varied from -1.75 degrees to 3.23 degrees, and so it can be approximated as being an overhead flyover with a fixed azimuthal angle of 0 degrees and a varying elevation emission angle. Figure 3 shows the spectrogram of the recorded flyover. Here, the rapid change in the Doppler shifted blade passage frequency is shown in each harmonic in the overhead region between 25-30 s.

\section{A. De-Dopplerization process}

The de-Dopplerization method from Greenwood and Schmitz ${ }^{7}$ was used to produce the de-Dopplerized signal shown by the blue trace in Figure 2. The blue trace is offset from zero to clearly show the original flyover recording. The red vertical lines represent the tracking boundaries of the recorded data that were used to obtain the deDopplerized signal. The de-Dopplerized blue trace in Figure 2 was obtained by back propagating the recording to a radius of 30.48 meters from the source. The spectrogram in Figure 4 shows that the de-Dopplerization process appears to roughly eliminate the effects of Doppler and spreading loss. The de-Dopplerization process as used here did not remove atmospheric absorption and terrain effects. Atmospheric absorption was assumed to be small because of the low frequency content of the signal. Ground and terrain effects were mitigated by using a recording location without significant terrain present and a microphone flush with the ground.

\section{B. Segmenting de-Dopplerized signal}

To apply the signal decomposition and synthesis equations from Section II, the full de-Dopplerized recording given by the blue trace in Figure 2 should be divided into contiguous segments. The time segments do not necessarily have to be of equal length. Figure 5 gives an example of segmenting the de-Dopplerized signal into 15 contiguous segments that are not equal in time length but are equal in angular arc length. This means that the range of emission angles from source to ground observer swept in each time segment is the same. In this case, the range is close to 11 degrees. The signal decomposition and synthesis equations in Section II are applied to each time segment separately. In Pera et al, the de-Dopplerized signal was used, but the segments were not contiguous. 


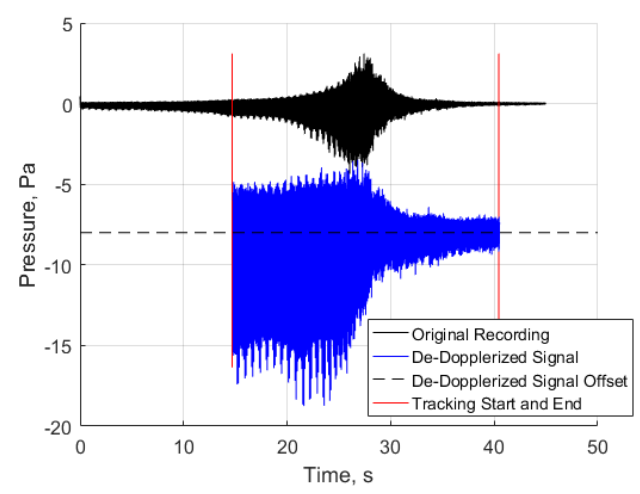

Figure 2. De-Dopplerized signal from original recording.

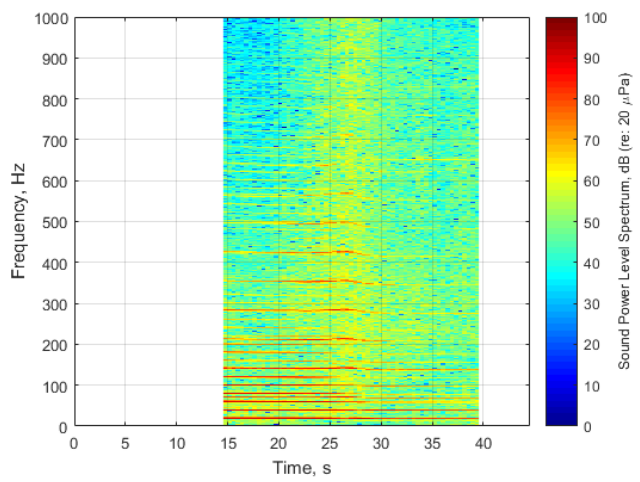

Figure 4. Spectrogram of de-Dopplerized recording.

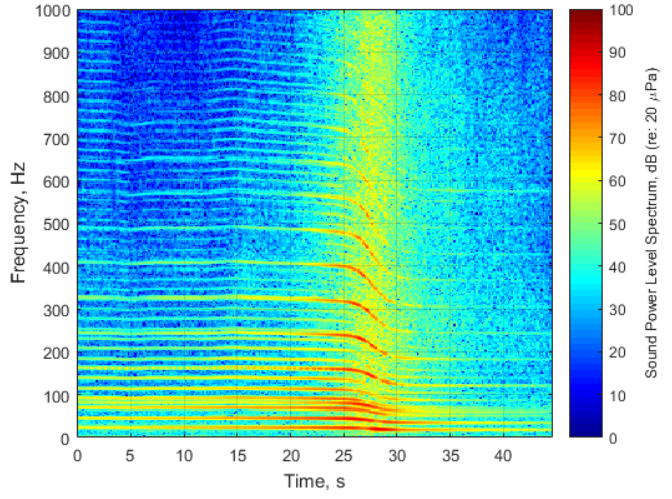

Figure 3. Spectrogram of original recording.

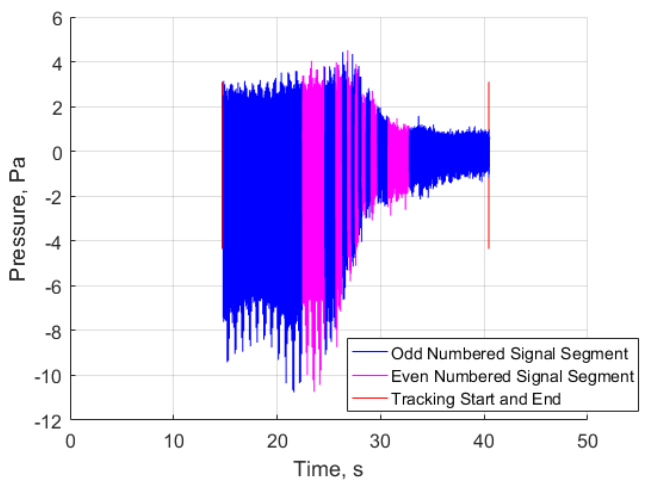

Figure 5. Segmentation of de-Dopplerized Signal

\section{Reconstruction of de-Dopplerized segments}

After the full de-Dopplerized signal is segmented into fifteen equal angular segments as shown in Figure 5, each segment can be reconstructed to test the signal decomposition process from Section II. A comparison of reconstructions with and without harmonic subtraction during signal decomposition is made here. Figure 6 shows the case without subtracting harmonics after their components are estimated. The example in Figure 6 is for the first of 15 segments from Figure 5. All subsequent figures in this section (Section III) and Section IV also use the first section from Figure 5 for their illustrations. The magenta trace in Figure 6 is the difference between the original and reconstructed signals. Visually, the reconstruction appears close to the original de-Dopplerized segment. The corresponding root mean square error (RMSE) between the reconstructed signal and the original is $13.6 \%$ of the root mean square (RMS) of the original signal. In contrast, the signal reconstruction in Figure 7 is computed when harmonics are subtracted, using the process indicated in Figure 1. The reconstructed and de-Dopplerized signals are visually indistinguishable. The RMSE between the reconstruction and the original signal reduced to $4.2 \%$ of the original signal RMS. It is because of this improvement that the component extraction method including subtraction of the reconstructed harmonics was adopted in this paper. Audible sound files of the reconstructed signal in Figure 6 and Figure 7, as well as the original de-Dopplerized signal for segment 1 of 15 are available for download. ${ }^{17}$

Further support for using the harmonic subtraction process is found by comparing sound quality metrics for the two reconstructions and the original de-Dopplerized segment, calculated here using the ArtemiS Suite from HEAD Acoustics. ${ }^{18}$ Values of sound quality metrics of loudness, tonality, impulsiveness, fluctuation strength, and roughness, for the reconstruction with subtraction were closer to those for the original de-Dopplerized signal than without subtraction. The sharpness values of the reconstructions are approximately equal and reduced slightly from the sharpness of the original signal. 


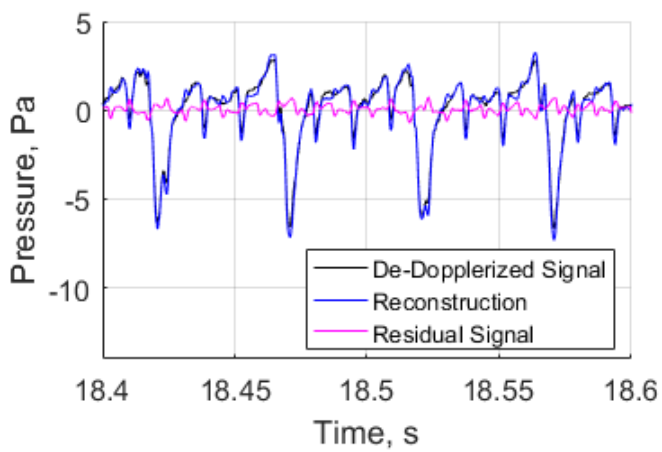

Figure 6. Reconstruction of a segment without subtraction.

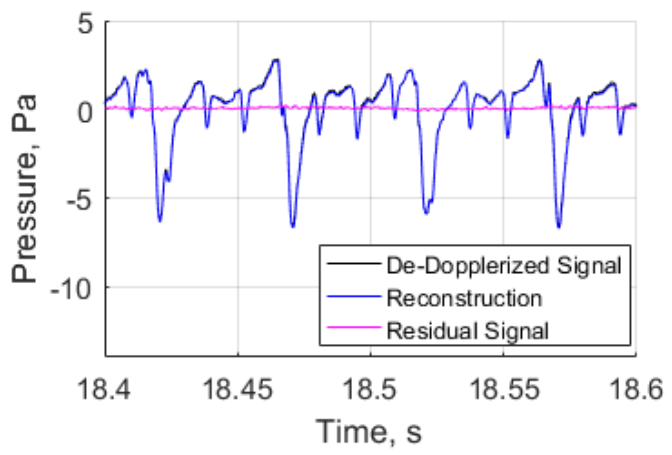

Figure 7. Reconstruction of a segment with subtraction.
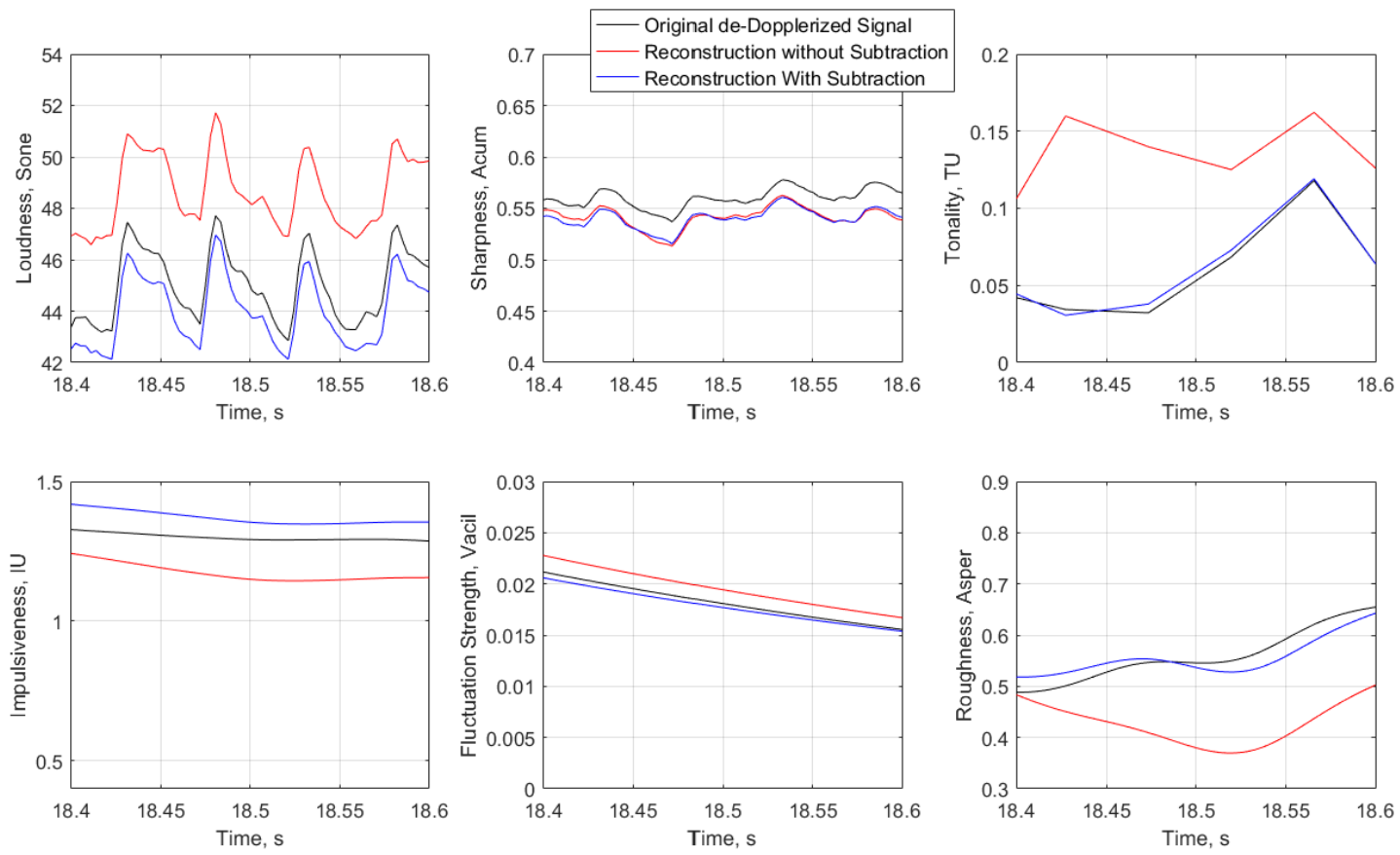

Figure 8. Sound quality metrics of reconstructed signals from a segment.

\section{Amplitude Modulation Model}

The amplitude modulation model used here was previously developed by Pera et al. ${ }^{11}$ and is described below. Although the model is identical to Pera et al., the results of its application are different due to the modifications to the signal decomposition and additive synthesis method described in Section II.

\section{A. Spectral characteristics of the amplitude modulation}

It is useful to examine the spectral characteristics of the harmonic amplitude modulations determined from the signal decomposition, which are the terms $\tilde{A}_{i, q}$ in Eq. (5), to guide the development of a model. Each harmonic of the main and tail rotors will have its own unique amplitude modulation spectrum. Figure 9 shows the sound pressure level of the amplitude modulation for the first three harmonics of the main rotor from the first segment of the deDopplerized signal in Figure 5. The roll off of the broadband spectra steepens above roughly $10 \mathrm{~Hz}$. In Pera et al., significant peaks appeared in the spectra due to leakage of neighboring tones into the passband of the filter used to isolate a particular harmonic. In this paper, these peaks are not as prominent in Figure 9, since the narrower $15 \mathrm{~Hz}$ bandpass filter isolated the harmonics, and the harmonics were subtracted after determining their components. As in Pera et al., spectra of the amplitude modulation from other recorded time segments are different in magnitude but similar in terms of their roll off behavior. 


\section{B. Band-limited random model of amplitude modulation}

Based on the above observations, it was decided to model the amplitude modulation as a band-limited random process. A $3^{\text {rd }}$-order Butterworth filter magnitude response curve was chosen as the archetype shape of the random process model. Such an approach was successfully used by Allen et al. ${ }^{9}$ to model turbofan tone modulations. An adaptation of that approach was made here, resulting in a model with two parameters: the cutoff frequency and a scale factor. The two parameters were individually determined for each main and tail rotor harmonic for each segment. With these two parameters, a time history of arbitrary duration of amplitude modulation data characteristic of each harmonic and emission angle could be generated. The process for determining the model parameters is briefly described next.

Starting with the sound pressure level spectrum of the amplitude modulation, a binary weighting vector was applied, which zeroed all frequency components following the first instance of the spectrum dropping $30 \mathrm{~dB}$ below the maximum value. The effect of that operation is seen in Figure 10. This weighting vector is useful for mitigating the impact of leakage from tones just outside the passband of the bandpass filter, such as the tone near $20 \mathrm{~Hz}$ in Figure 9. The RMS difference between the weighted sound pressure level spectrum and the magnitude response curve was minimized as a function of a cutoff frequency. The resulting filter was applied to white noise of arbitrary duration, and a scale factor was found to match the level of filtered noise spectrum with the amplitude modulation spectrum up to the cutoff frequency.

A sample spectrum of the output of the amplitude modulation model is shown in Figure 11 for the third harmonic of the main rotor from a recording segment. The roll off and spectrum level of the filtered signal closely matches those of the original amplitude modulation. The cutoff frequency for most of the main and tail rotor harmonics lies in the range of 5-12 Hz, as shown in Figure 12. The range of scale factors for main and tail rotor harmonics is shown in Figure 13. No attempt was made to model the cutoff frequencies and scale factors from observed statistics.

The effectiveness of the amplitude modulation model was assessed by reconstructing the original de-Dopplerized signal segment. Here the amplitude modulation data was provided by the model instead of from the signal decomposition process, that is,

$$
p_{q}(t)=\sum_{i=1}^{N, M}\left[\bar{A}_{i, q}+\tilde{A}_{i, q}^{\text {model }}\right] \cos \left(2 \pi \bar{f}_{i} t+\tilde{\phi}_{i, q}+\phi_{o, i, q}\right) .
$$

This form of the synthesis equation still uses the phase modulation data obtained from the signal decomposition process. The reconstructed waveform is shown by the orange trace in Figure 14, and the associated sound is provided as an audio file. ${ }^{12}$ The RMSE between the reconstruction with the amplitude modulation model and the original deDopplerized segment was $21.5 \%$ of the RMS of the original de-Dopplerized segment. Reconstructions of other segments gave comparable results, indicating that this model of the amplitude modulation is also effective at other emission angles.

If the phase modulation data are removed from Eq. (9), the result is the green trace in Figure 14. The RMSE without the phase modulation increases to $54.4 \%$ of the original de-Dopplerized signal RMS. This signal without the phase modulation data is also provided as an audio file. ${ }^{12}$

The amplitude modulations can be extended indefinitely in time. In Figure 15, the reconstructed signal is extended beyond the original de-Dopplerized signal with an amplitude modulation model but with no phase modulation. Several attempts were previously made to model the phase modulations. ${ }^{19}$ Unfortunately, all methods produced audible artifacts that often diminished the perceived impulsiveness. Although the aperiodic phase components for a recorded segment duration were extracted and helped produce the reconstructions in Figure 6, Figure 7, and Figure 14, they could not be extended indefinitely in time. Other possible approaches to extend the phase modulation have not yet been exhausted, and investigation into them will continue as future work. Flyover auralizations are therefore currently performed without the benefit of phase modulation in the source noise synthesis.

A comparison of the sound quality metrics for the reconstructions is shown in Figure 16. Metrics derived from the reconstruction with extracted amplitude and phase modulations data, shown in the blue trace, serve as the basis for comparisons. Note that the blue trace in Figure 16 is identical to the blue trace in Figure 8 for reconstruction with harmonic subtraction during signal decomposition. In addition to the metrics derived from reconstructions with modulation, the red trace in Figure 16 shows the sound quality metrics for a periodic reconstruction involving just $\bar{A}$ and $\phi_{o}$, that is, without modulations.

A primary conclusion from Figure 16 is that the phase modulations significantly impact impulsiveness. The impulsiveness values of the reconstruction with the amplitude modulation model and phase modulation data, given by the orange trace, are significantly lower than values for reconstruction that includes both the amplitude and phase modulation data. When the reconstruction excludes phase modulation, shown by the green trace, the impulsiveness and roughness are drastically reduced, dropping to the same level as the periodic reconstruction. Phase heavily 
influences impulsiveness, and its absence may be one reason for the reduced impulsiveness levels. Roughness also appears to be significantly affected by the exclusion of phase modulation, but this result is not surprising since Krishnamurthy et al., ${ }^{20}$ found roughness and impulsiveness to be highly dependent for helicopter-like sounds based on a recording of an AS350 helicopter.

The values of other metrics are not as drastically affected in Figure 16 as the impulsiveness and roughness values. Sharpness, tonality, and fluctuation strength have roughly the same levels, though not identical, between the reconstruction with amplitude and phase modulation data and the other three reconstructions. Although the loudness values differ among the traces, these differences were not consistently seen in all segments of the recording.

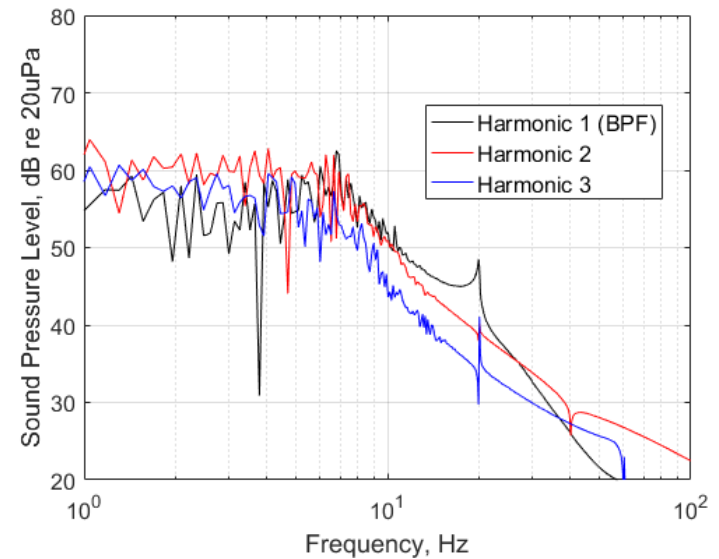

Figure 9. Sound pressure level of the main rotor amplitude modulations from a segment.

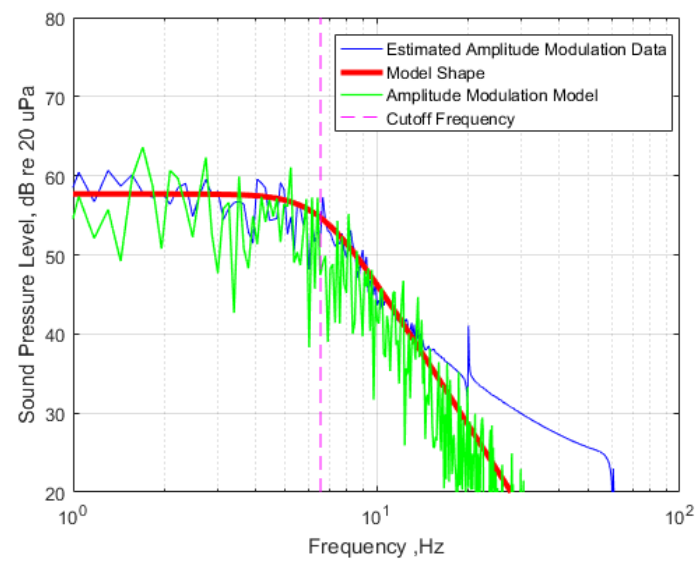

Figure 11. Sound pressure level spectrum of modeled modulation data compared with original and filter fit for $3^{\text {rd }}$ main rotor harmonic of a segment.
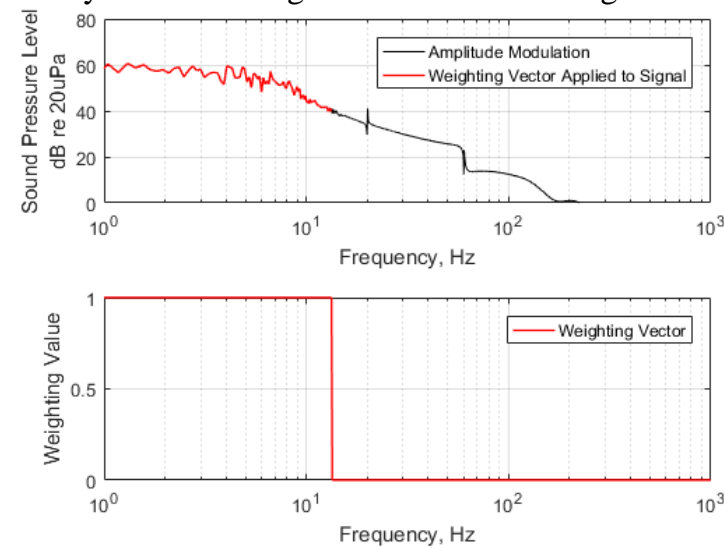

Figure 10. Sound pressure level of the $3^{\text {rd }}$ main rotor harmonic amplitude modulation and its weighting vector for a segment.

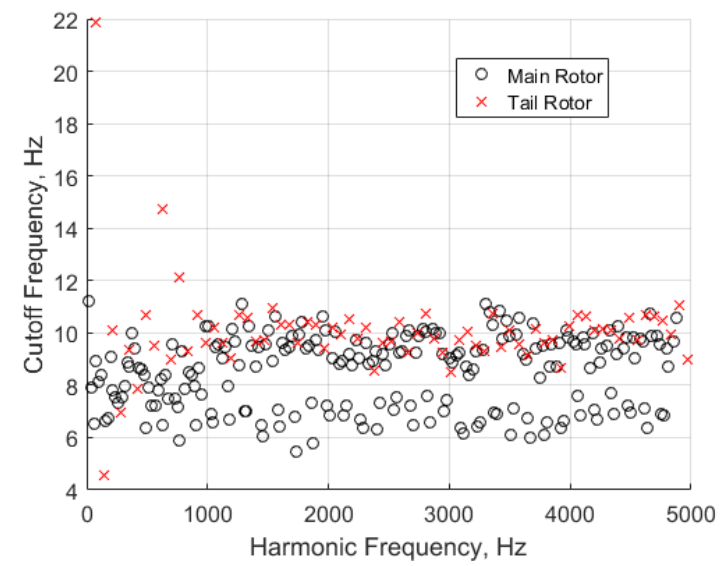

Figure 12. Main and tail rotor cutoff frequencies for harmonics of a segment. 


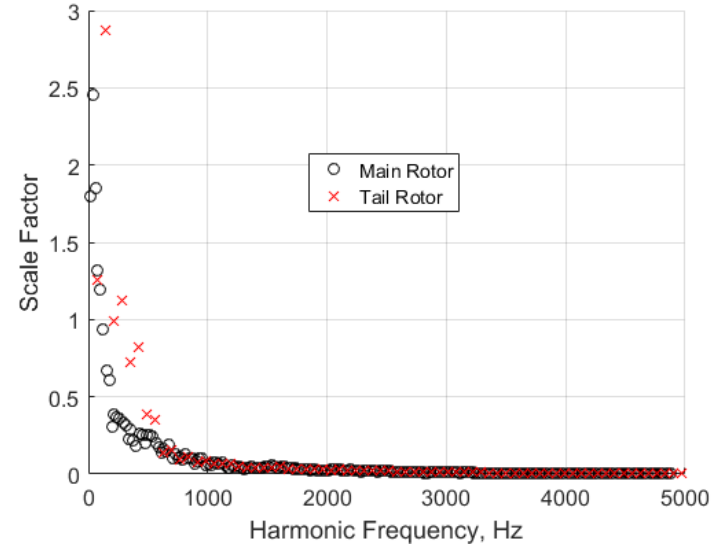

Figure 13. Main and tail rotor scale factors for harmonics of a segment.

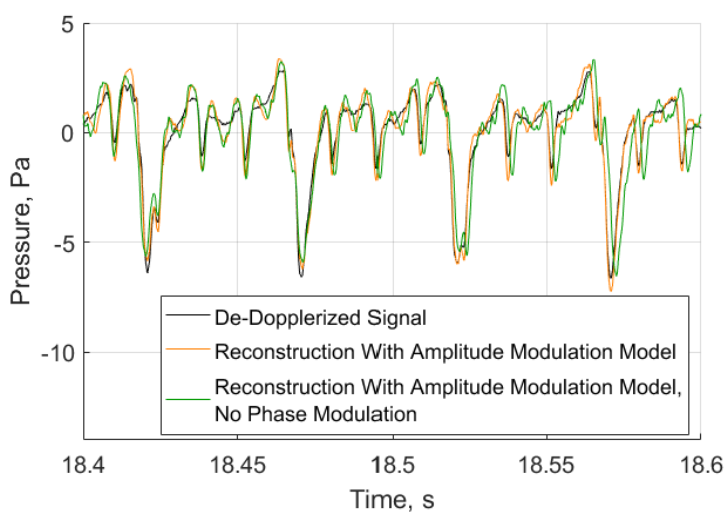

Figure 14. Original and reconstructed signals using modeled amplitude modulation for a segment.

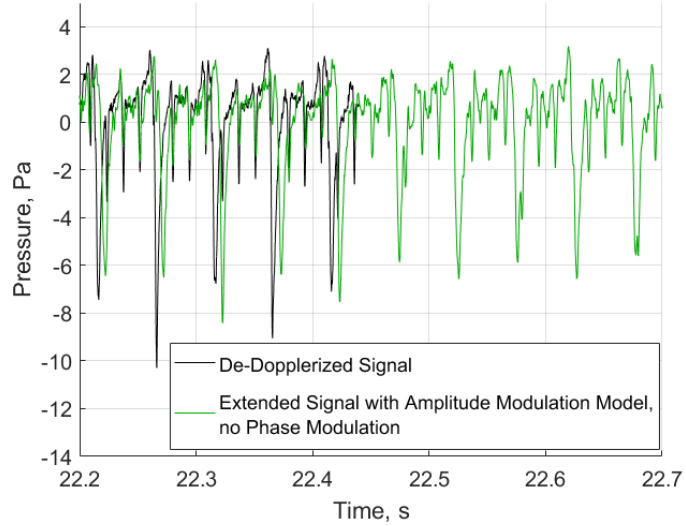

Figure 15. Extending reconstruction beyond original signal. 

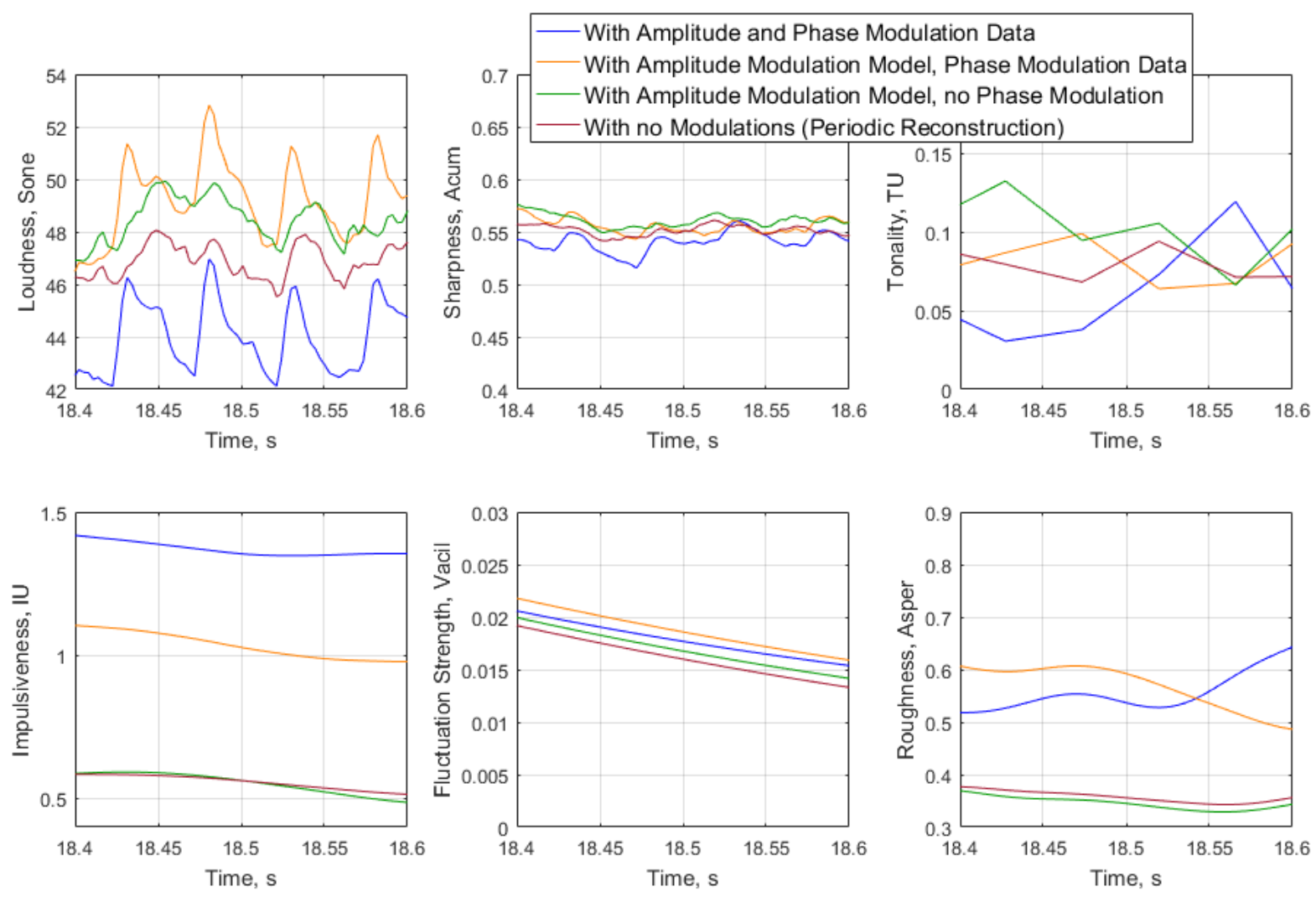

Figure 16. Sound quality metrics of a reconstructed segment with amplitude modulation model.

\section{Auralization of a Flyover}

In order to auralize a flyover event, the noise of the moving source must be synthesized and that noise must be propagated to an observer on the ground. The synthesis process for a moving source and stationary observer is described below. The auralizations demonstrated in this paper will attempt to recreate the original overhead helicopter flyover using the tracking data from the flyover recording. For the case of an overhead flyover, the dependency on azimuthal angle vanishes. The tracking data were previously used to de-Dopplerize the recording. Hence, the synthesis and auralization times in this paper will be as long as the de-Dopplerized signal. The sample rate for the synthesis and auralization of the flyover was $44.1 \mathrm{kHz}$. As discussed in the previous section, the synthesis process does not currently account for phase modulation.

\section{A. Source noise synthesis of a moving source}

Synthesis of the moving source noise is analogous to what a roving microphone would record at some fixed reference distance from the source. The synthesis equation of the moving source is written as

$$
p(\theta, t)=\sum_{i=1}^{N, M}\left[\bar{A}_{i}(\theta)+\tilde{A}_{i}(\theta, t)\right] \cos \left(2 \pi \bar{f}_{i} t+\phi_{o, i}(\theta)\right)
$$

in which the dependence of $\theta$ on $t$ is implicit. In the above, the phase modulation term has been omitted. Although the frequency of each harmonic, $\bar{f}_{i}$, can in principle also change with angle, it is fixed for all emission angles and time in this paper as described in Section II. As discussed in the preceding sections, the amplitude and phase terms for the source have been determined at discrete emission angles corresponding to the segmentation of a flyover recording. The next section describes how source characteristics at those discrete emission angles are used to determine source characteristics at the intermediate emission angles needed to recreate the flyover. 


\section{B. Interpolation}

\section{Interpolation of periodic data}

During the course of the flyover, the emission angle between the vehicle and a stationary observer is evaluated at every sample time. The periodic data needed in Eq. $(10),\left(\bar{A}, \phi_{o}\right)$, are determined by interpolating between the discrete emission angles associated with each segment of the flyover recording. In this paper, spherical interpolation was used to interpolate the initial phase to avoid potential artifacts in the auralized sound, as described by Krishnamurthy et al. $^{21}$

\section{Interpolation of amplitude modulation model}

Data representative of the amplitude modulation for a flyover were synthesized using an overlap-add technique similar to that implemented by Allen et al. ${ }^{9}$ to synthesize broadband noise. The hop interval, or filter update rate, for the overlap-add approach was set to 512 samples $(11.6 \mathrm{~ms})$. The cutoff frequency and scale factor of the amplitude modulation model for each harmonic were interpolated at the emission angles corresponding to the hop intervals. Because the mean cutoff frequency of the amplitude modulation models was about $10 \mathrm{~Hz}$ for the main and tail rotor harmonics, the length of the pressure time histories generated using the amplitude modulation model was set to two seconds, or $88.2 \mathrm{k}$ samples, corresponding to a frequency resolution of $0.5 \mathrm{~Hz}$. The pressure time histories were independently synthesized off-line for each of the main and tail rotor harmonics for the entire flyover. Samples were subsequently drawn from the amplitude modulation model one at a time in the calculation of the total flyover pressure time history, Eq. (10).

\section{Synthesized Sound and Propagation}

The interpolation process for the periodic and amplitude modulation just described was applied to the 15 deDopplerized segments from Figure 5. The blue trace in Figure 17 shows the resulting synthesized signal. It is offset from zero to more clearly show the original de-Dopplerized signal for comparison.

Using the tracking data and NASA Auralization Framework (NAF), the synthesized signal in Figure 17 can be propagated to a ground observer during an overhead flyover to create an auralization. The original de-Dopplerized signal did not remove atmospheric absorption, therefore, the NAF propagation did not add atmospheric absorption. However, the de-Dopplerization process did account for the pressure doubling at the ground plane microphone, so the NAF propagation specified an observer on an infinitely hard ground to simulate that pressure doubling. The blue trace in Figure 18 shows the propagated synthesized sound compared with the original recording. The comparison is very good, and ways to address the slight deviations, especially at the start of the flyover, will be discussed shortly. The propagated signal trace in Figure 18 is available as an audio file. ${ }^{17}$

In this paper, the initial phases of segments were aligned as described in Section II.B. If this alignment is not done, there is a noticeable effect, as shown in Figure 19. Compared to the trace in Figure 18, there is a visible difference between the original and synthesized signals near the 23 second mark.

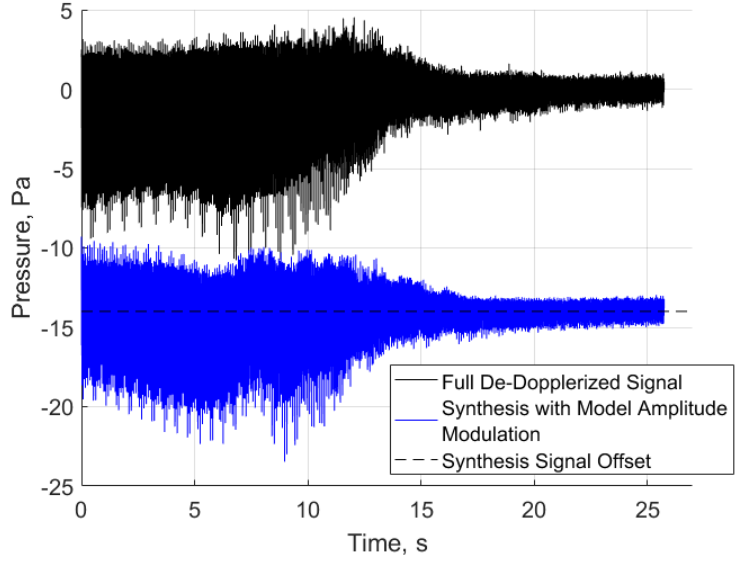

Figure 17. Synthesized signal using 15 equal angle segments.

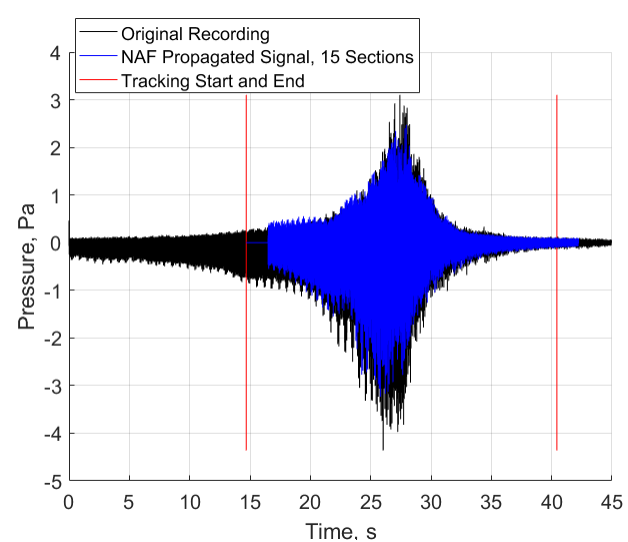

Figure 18. Propagation of synthesized sound using 15 segments. 


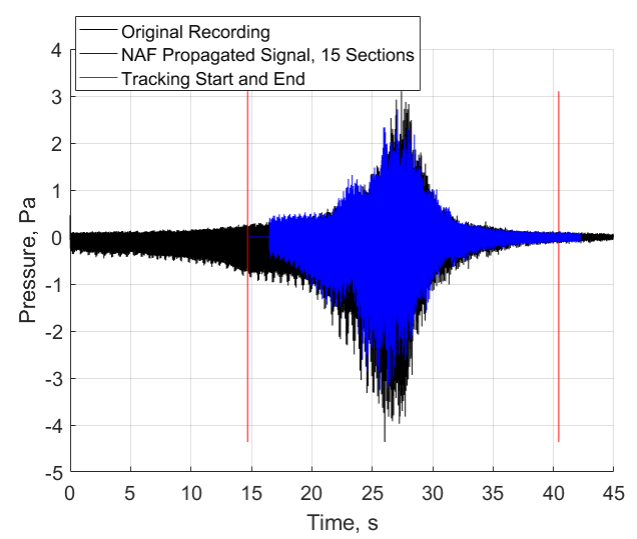

Figure 19. Synthesis with phase misalignment.

\section{Changing the Number of Segments}

Two methods of segmenting the de-Dopplerized flyover data for determining the angle-dependent source characteristics are next explored. In the first approach, an equal angle segmentation is considered. In the second approach, an equal time segmentation is undertaken. Increasing the number of segments of the original deDopplerized signal from 15 to 30 segments reduces the segment angle span to 5.5 degrees. The resulting segmentation is shown in Figure 20. The auralization corresponding to this segmentation, inclusive of all steps in the process, produces the blue signal shown in Figure 21. The discrepancy from the original recording at the beginning of the flyover appears to be mitigated compared to Figure 18, but there appears to be greater discrepancy in the middle of the flyover. With an increase to 30 equal angle segments, the segments in the middle of the recording are of shorter duration, and signal transition artifacts from the bandpass filtering have a larger contribution to an extracted harmonic relative to signal length. Estimates of harmonic components therefore contain more error. The propagated signal in Figure 21 is available as an audio file. ${ }^{17}$

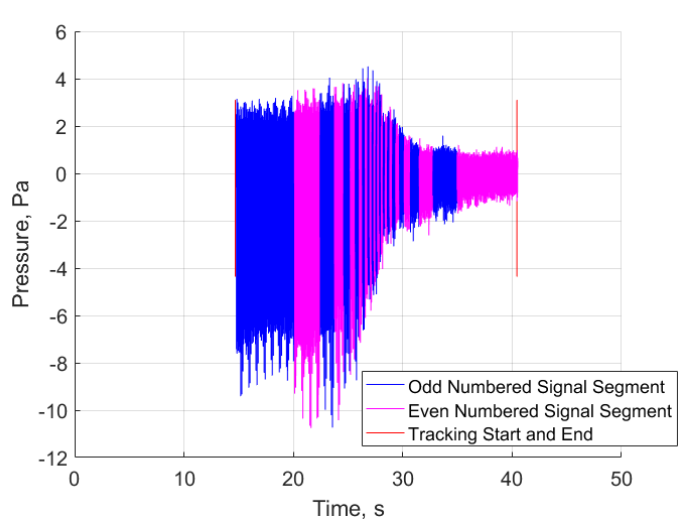

Figure 20. Segmenting de-Dopplerized signal into 30 segments.

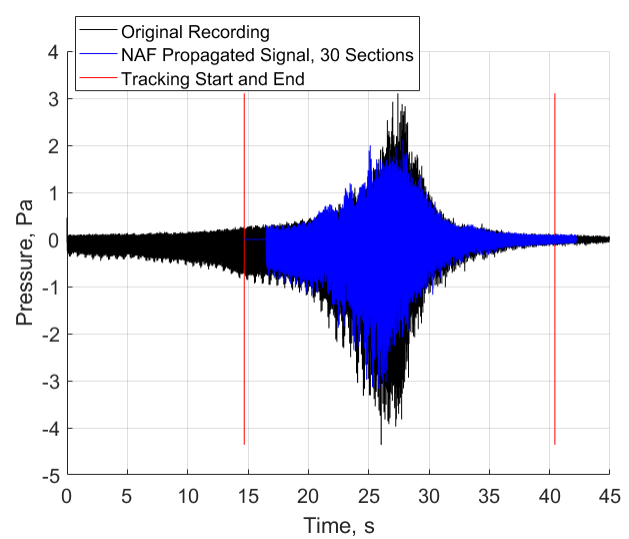

Figure 21. Propagation of synthesized sound using 30 segments.

Instead of dividing the de-Dopplerized signal into equal angle segments, we can alternatively divide the signal into equal time segments. Figure 22 shows the de-Dopplerized signal divided into 26 segments of one second each. The NAF propagation output with these 26 segments is shown in Figure 23. The middle of the flyover has a closer fit to the original recording but there appear to be a few pressure spikes at the beginning of the flyover. The propagated signal trace in Figure 23 is available as an audio file. ${ }^{17}$ 


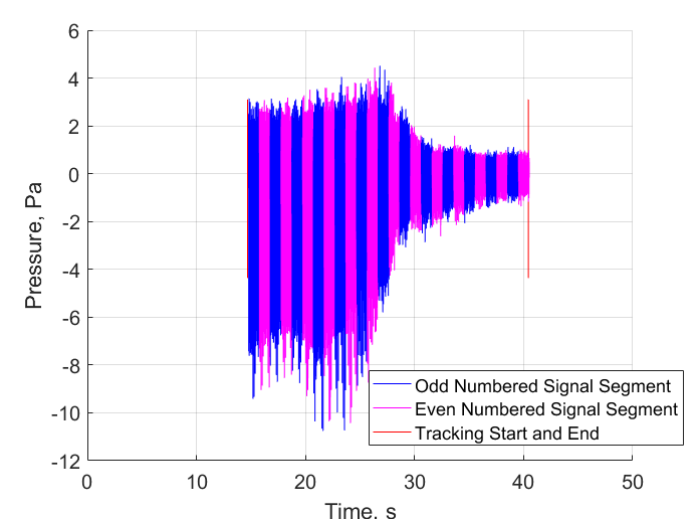

Figure 22. Segmenting de-Dopplerized signal into 26 equal time segments.

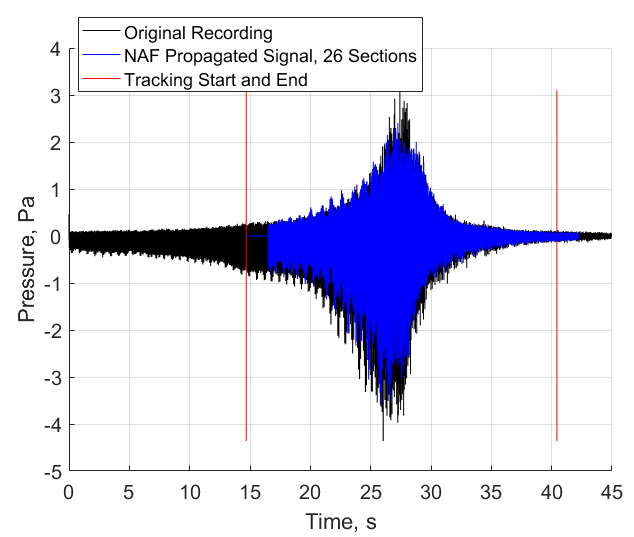

Figure 23. Propagation of synthesized sound using 26 equal time segments.

In Figure 24, the blue trace gives the angular length of each of the 26 equal time sections. The middle sections have a larger emission angle length since the emission angle changes faster when the flyover is directly above the ground observer. Both the left and right graphs in Figure 24 express the same information, but the $\mathrm{x}$-axis on the left graph is the section number, while the $\mathrm{x}$-axis on the right graph is the emission angle at the center of each section. The black trace is the emission angle length of each of the 15 sections from Figure 5. The red trace is the emission angle length of each of the 30 sections from Figure 20.

An improved method to divide the de-Dopplerized recording may be to use a hybrid segmentation scheme in which equal angle segments are selected at the beginning and end of the recording and equal time segments are selected in the middle. Figure 24 can provide useful guidance to such a hybrid segmentation. Segments below the horizontal black and red traces can be of equal angular length, and segments above the horizontal traces can be of equal time length. Developing an improved scheme to segment the recording will be investigated as future work.

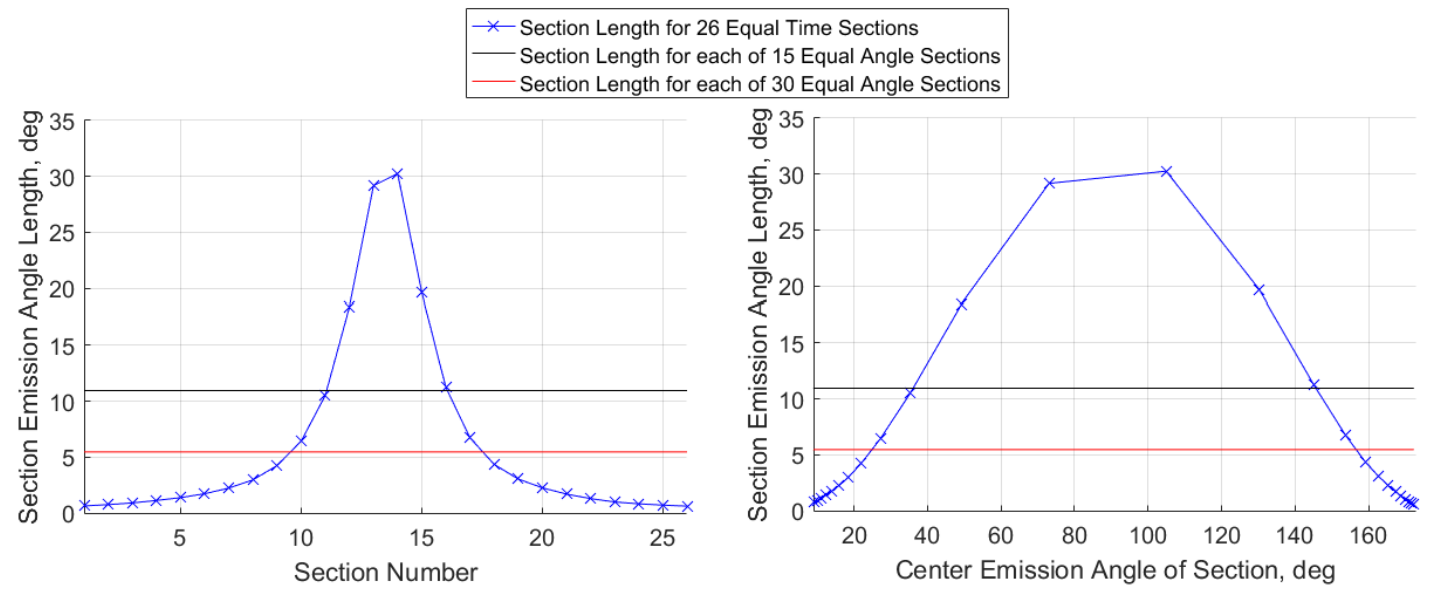

Figure 24. Emission angle length for each equal time section.

\section{E. Sound Quality Metrics of a Flyover}

Sound quality metrics of the original recorded signal are next compared with metrics computed from two flyover auralizations using the 26 equal time segment data. One auralization omitted any amplitude modulation data, i.e., only the periodic components were utilized. The other auralization used the modeled amplitude modulation with equal time segments, as shown in Figure 23. The trends in the sound qualities for auralizations with and without amplitude modulation shown in Figure 25 resemble the trends contained in Figure 16. Impulsiveness and roughness show the most drastic differences followed by loudness between the original recording and the auralizations. The difference in sharpness between the recording and auralizations is likely due to higher harmonics being present in the original recording. Since sharpness levels between the synthesized sounds and the original de-Dopplerized signal in Figure 16 are similar, sharpness of the auralizations will probably not improve beyond what is given in Figure 25. Since sharpness is related to loudness, the loudness discrepancy between the recording and auralizations may not completely 
disappear, but extending phase modulation along with amplitude modulation might mitigate the difference. Extending phase modulation is expected to lower the differences for impulsiveness and roughness, as was the case for the source noise synthesis in Figure 16. Consequently, it is critical that methods to extend the phase modulation indefinitely be found so that auralizations suitable for assessments of community response can be produced.
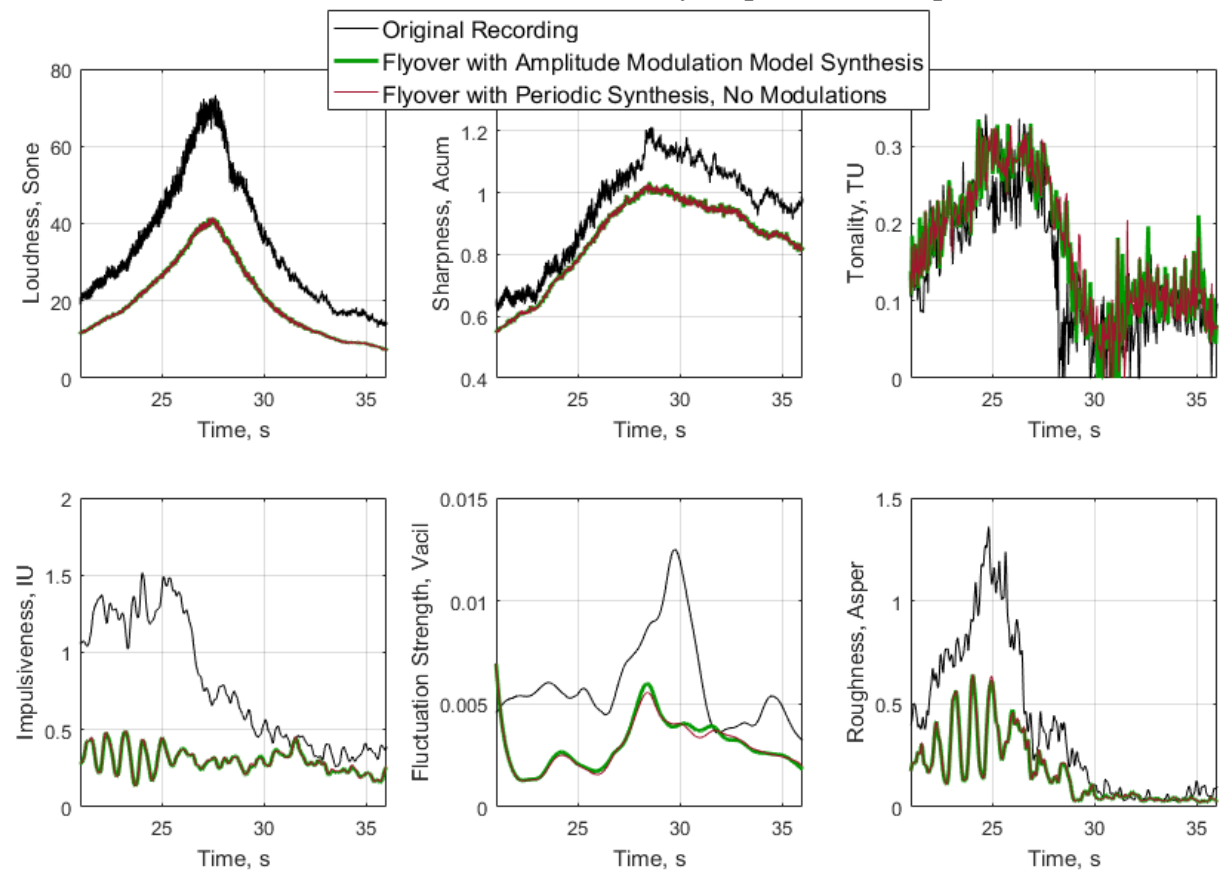

Figure 25. Comparison of sound quality metrics for a flyover auralized using 26 segments with those from the original recording.

\section{Conclusion}

The present work extends previous work for auralizing a helicopter flyover based on a segmented, de-Dopplerized recording of an actual helicopter flyover. Contiguous de-Dopplerized time segments of the recording of an AS350 helicopter were used to determine source noise characteristics as a function of source emission angle. Starting with the results of Pera et al., ${ }^{11}$ an amplitude modulation model was extended indefinitely in time. An improvement from Pera et al. was made in the signal decomposition process by subtracting rotor harmonics from the original recording at each segment as their components were estimated. The subtraction method reduced the RMSE between the reconstructed signal and the original recording and also brought the sound quality of the reconstruction closer to the recording. Combined with a smaller $15 \mathrm{~Hz}$ bandpass filter to isolate harmonics, the subtraction method also mitigated peaks due to leakage in the amplitude modulation spectrum. An overlap-add method was used to produce amplitude modulation data for the full flyover. As an improvement to handling the synthesized initial phase from Pera et al., phase alignment and spherical interpolation were used. Auralizations using different numbers and lengths of segments of the recording were generated in order to understand which segmentation scheme produces the closest result to the original recording. A hybrid segmentation was proposed, whereby distant portions of the flyover are divided into equal angles and close portions of the flyover, when the vehicle is nearly overhead, are divided into equal time segments. It was also demonstrated that excluding phase modulation from the auralization significantly reduces the impulsiveness. Hence it is critical in future work to develop a phase modulation model.

\section{Acknowledgments}

This work was performed with support from the Revolutionary Vertical Lift Technology Project of the NASA Advanced Air Vehicles Program. 


\section{References}

1"Report on the Los Angeles Helicopter Noise Initiative," Federal Aviation Administration 2013.

2"The New York North Shore Helicopter Route," Federal Aviation Administration, Department of Transportation, 14 CFR Part 93, Docket No. FAA-2010-0302, 2010.

${ }^{3}$ Basner, M., Clark, C., Hansell, A., Hileman, J., Janssen, S., Shepherd, K., and Sparrow, V., "Aviation Noise Impacts: State of the Science," Noise and Health, Vol. 19, No. 87, 2017, pp. 41-50.

${ }^{4}$ Vorländer, M., Auralization - Fundamentals of acoustics, modelling, simulation, algorithms and acoustic virtual reality. SpringerVerlag, Berlin, 2008.

${ }^{5}$ Rizzi, S.A. and Christian, A., "A method for simulation of rotorcraft fly-in noise for human response studies," InterNoise 2015, San Francisco, CA, 2015.

${ }^{6}$ Rizzi, S.A., Christian, A.W., and Rafaelof, M., "A laboratory method for assessing audibility and localization of rotorcraft fly-in noise," To appear in AHS Journal, Vol., No. 2019.

${ }^{7}$ Greenwood, E. and Schmitz, F.H., "Separation of main and tail rotor noise from ground-based acoustic measurements," AIAA Journal of Aircraft, Vol. 51, No. 2, 2014, pp. 464-472.

${ }^{8}$ Hardwick, J.R., "Synthesis of rotorcraft noise from flyover data," M.S. Thesis, Department of Mechanical Engineering, Virginia Tech, Blacksburg, VA, 2014.

${ }^{9}$ Allen, M.P., Rizzi, S.A., Burdisso, R., and Okcu, S., "Analysis and synthesis of tonal aircraft noise sources," 18th AIAA/CEAS Aeroacoustics Conference, AIAA-2012-2078, Colorado Springs, CO, 2012.

${ }^{10}$ Hardwick, J.R., Christian, A., and Rizzi, S.A., "Evaluation of the perceptual fidelity of a novel rotorcraft noise synthesis technique," The Journal of the Acoustical Society of America, Vol. 136, No. 4, 2014, pp. 2287-2287.

${ }^{11}$ Pera, N.M., Rizzi, S.A., Krishnamurthy, S., Fuller, C.R., and Christian, A.W., "Development of a method for analysis and incorporation of rotorcraft fluctuation in synthesized flyover noise," AIAA SciTech 2018, AIAA-2018-0267, Kissimmee, FL, 2018.

${ }^{12}$ Aumann, A.R., Tuttle, B.C., Chapin, W.L., and Rizzi, S.A., "The NASA Auralization Framework and plugin architecture," InterNoise 2015, San Francisco, CA, 2015.

${ }^{13}$ Stephenson, J.H. and Greenwood, E., "Effects of vehicle weight and true versus indicated airspeed on BVI noise during steady descending flight," AHS Forum 71, Virginia Beach, VA, 2015.

${ }^{14}$ Watts, M.E., Greenwood, E., Sim, B., Stephenson, J.H., and Smith, C.D., "Helicopter acoustic flight test with altitude variation and maneuvers," NASA TM-2016-219354, 2016.

${ }^{15}$ Kirkpatrick, S., Gelatt, C.D., and Vecchi, M.P., "Optimization by simulated annealing," Science, Vol. 220, No. 4598, 1983, pp. 671-680.

${ }^{16}$ Farassat, F., "Derivation of formulations 1 and 1A of Farassat," NASA TM-2007-214853, 2007.

17"Aircraft flyover simulation," https://stabserv.larc.nasa.gov/flyover/, NASA, 2018.

18"ArtemiS Suite," 12.2, HEAD acoustics GmbH, NVH Division, 2016.

${ }^{19}$ Pera, N.M., "Development of a method for analysis and incorporation of rotorcraft fluctuation in synthesized flyover noise," M.S. Thesis, Department of Aerospace and Ocean Engineering, Virginia Tech, Blacksburg, VA, 2017.

${ }^{20}$ Krishnamurthy, S., Christian, A.W., and Rizzi, S.A., "Psychoacoustic test to determine sound quality metric contributions to rotorcraft noise annoyance," InterNoise 2018, Paper 1338, Chicago, IL, 2018.

${ }^{21}$ Krishnamurthy, S., Rizzi, S.A., Boyd Jr., D.D., and Aumann, A.R., "Auralization of rotorcraft periodic flyover noise from design predictions," 74th AHS Forum, Paper 166, Phoenix, AZ, 2018. 